\title{
An Automated Process for Inclusion of Package Dies and Circuitry within a Textile Yarn
}

\author{
Dorothy Hardy \\ Advanced Textiles Research Group \\ Nottingham Trent University \\ dorothy.hardy@ntu.ac.uk \\ Carlos Oliveira \\ Advanced Textiles Research Group \\ Nottingham Trent University \\ jose.oliveira@ntu.ac.uk \\ John Tudor \\ Electronics and Computer Science \\ University of Southampton \\ mjt@ecs.soton.ac.uk \\ Tilak Dias \\ Advanced Textiles Research Group \\ Nottingham Trent University \\ tilak.dias@,ntu.ac.uk
}

\author{
Ioannis Anastasopoulos \\ Advanced Textiles Research Group \\ Nottingham Trent University \\ ioannis.anastasopoulos@ntu.ac.uk
}

\author{
Theodore Hughes-Riley \\ Advanced Textiles Research Group \\ Nottingham Trent University \\ theodore.hughesriley@ntu.ac.uk \\ Russel Torah \\ Electronics and Computer Science \\ University of Southampton \\ rnt@ecs.soton.ac.uk
}

\author{
M-Nour Nashed \\ Advanced Textiles Research Group \\ Nottingham Trent University \\ m-nour.nashed2014@my.ntu.ac.uk \\ Abiodun Komolafe \\ Electronics and Computer Science \\ University of Southampton \\ A.O.Komolafe@soton.ac.uk \\ Steve Beeby \\ Electronics and Computer Science \\ University of Southampton \\ spb@ecs.soton.ac.uk
}

\begin{abstract}
The integration of small electronic components into textile fabrics, without compromising the textile qualities such as flexibility and conformability, is necessary in ensuring wider adoption of electronic textiles. A solution is to use flexible, electronic yarns that incorporate electronic components within the fibers of the yarn. The production of these novel yarns was initially a craft skill, with inclusion of electronics within each section of yarn taking 60 - 90 minutes. A prototype, automated production process was developed to speed up the manufacturing process to 6 minutes. This paper describes the process, using machinery and methods from both electronics and textiles applications.
\end{abstract}

Keywords - automation, electronic textiles, package dies, smart textiles

\section{INTRODUCTION}

The growing market for electronic textiles is predicted to be 'approaching \$5bn by 2027' [1] showing that there is a desire to place electronics within clothing, in close contact with the human body [2] to create interactive garments. Satisfying this market for smart textiles and wearables will require integration of electronics into fabrics, whilst maintaining the ability of the textiles to drape and conform to the body. A solution is to use flexible electronic yarns (E-yarns) that incorporate electronic components such as multi-terminal package dies within the yarn structure [3]. An alternative is to attach circuitry to flexible, heat-resistant Kapton strip [4] so that more complex circuitry can be integrated within a yarn structure. The resulting E-yarns can be incorporated into electronic textiles using knitting, weaving or embroidery processes. The aim is to ensure that the electronics take up minimal space, and the entire construction is as flexible as possible, so that the components can then be included within a textile construction that can drape and conform.

LEDs within E-yarns give the opportunity to create flexible clothing and costumes, as well as advertising the existence of the E-yarn, as the LEDs are visible due to their illumination. Applications for E-yarns include illuminated garments [5], costumes for performance, and temperature measurement in socks [6].

Standard circuits are often built up on printed circuit boards (PCBs) [7], [8], including flexible PCBs [9]. PCBs provide a stable platform for attachment of dies and connections. The process of manufacture of E-yarns is similar to that of producing a $\mathrm{PCB}$, but without a rigid, board on which soldering can take place: construction of E-yarn takes place using flexible components without attachment to a rigid substrate.

E-yarn construction required the development of new manufacturing processes that included the use of textiles and electronics materials and techniques. The novelty of the process was in:

1) The method of mixing both textiles and electronics materials and techniques;

2) The creation of a micropod around the electronics to make the construction chemically-resistant and washable;

3 ) The incorporation of the electronics within a textile yarn.

Project funded by EPSRC grant EP/M015149/1 and Nottingham Trent University. 
Initially, production of LED E-yarns was a time-consuming craft skill [10]. Automation of the process was required in order to manufacture E-yarns on a scale compatible with the demand for textiles containing electronics.

\section{A. Aim}

The aim of this work was to develop a working prototype process for manufacture of E-yarns: This could then be taken up by manufacturing companies. The purpose was not to produce the most cost-effective manufacturing solution, as it was realised that later iterations of the design would bring the cost down. The initial goal was to automate the process within a six-month timeframe, so that the production speed of E-yarns could be increased. LED yarn manufacture was chosen as the example. Other, small package dies can also be incorporated into E-yarn using the method described, and the process can be adapted for attachment of circuitry on Kapton strip to copper wire.

\section{LED YARN MATERIALS AND CONSTRUCTION}

LED yarns contain LEDs attached to a conductive core, within a flexible yarn structure. Fig 1 shows an illuminated LED yarn and Fig 2 shows three stages in the creation of an Eyarn containing a package LED, in diagrammatic form. The central, conductive wire is required to be flexible and also resistant to breakage during repeated flexing of the textile construction. A multi-strand copper wire (7-strand copper wire with $50 \mu \mathrm{m}$ strand diameter: Knight Wire, Potters Bar, UK) was chosen, as this was more flexible than a single strand wire of a similar thickness. Two-terminal LEDs were soldered onto the wire in series (more complex yarn constructions would require use of insulated wires running in parallel.) Fig 2a shows a two-terminal LED attached to the wire. The chosen LEDs (Kingbright KPHHS-1005SURCK Red LED, 630 nm 1005 (0402), Rectangle Lens SMD package: RS components, Corby, UK) were $1 \times 0.5 \times 0.3 \mathrm{~mm}$ : a size that could be handled by the available machinery. Fig $2 \mathrm{~b}$ shows a carrier yarn running parallel to the copper wire, and a resin micropod that protects the solder joints as well as preventing moisture ingress into the die. The micropod also attached the carrier yarn to the construction, minimizing tensile forces being transmitted to the copper wire and solder joints. A Vectran ${ }^{\mathrm{TM}}$ multifilament yarn (Kururay, Tokyo, Japan) was chosen to provide the necessary tensile strength. The resin micropod was made from Dymax Multi-cure ${ }^{\circledR}$ 9001-E-V3.5 Resilient, (Intertronics, Kidlington, UK) which is a clear polymer encapsulant. This could be applied in small quantity using a mechanized fluid dispenser, then UV-cured within a few seconds, to enable rapid, automated manufacture.

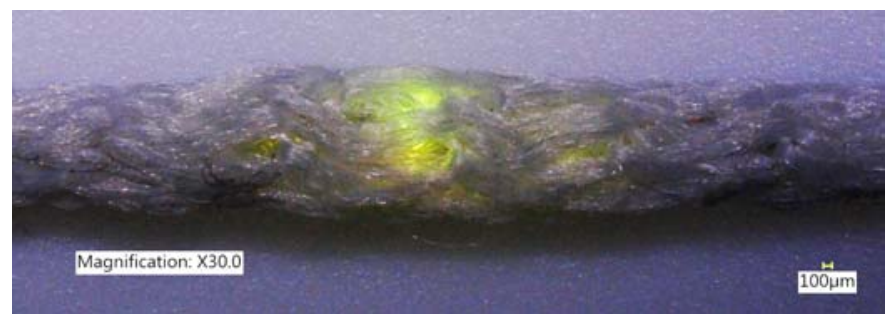

Fig. 1. An E-yarn containing an LED, shown at $30 \mathrm{x}$ magnification
Fig 2c shows the outer, knitted E-yarn structure that provides the required textile softness. Additional textile yarns were twisted around the wire before adding the knitted, outer structure. These yarns held the copper wire in place, preventing it from protruding through the knitted sheath. Additional packing fibers were also contained within the completed E-yarn structure, in order to fill out the outer, knitted tube. This prevented the structure from being a flat tube with bulges where the micropods were located, giving normality to the Eyarn.

\section{METHOD}

The original, craft process for fabrication of E-yarn required four stages. Each of these stages was automated separately with a longer-term aim of bringing the processes together to create one, continuous production line for E-yarn. An iterative design process was implemented using 'forward creation with mental leap' [11]. Machinery from both textiles and electronics processing applications were used. The stages of manufacture of an E-yarn containing an LED are described below, but the process can be applied to other package dies such as thermistors [6] and to circuitry on flexible Kapton strip.

\section{A. I. Soldering the die to wire}

A two-terminal LED package die was soldered onto a copper wire. This process required accurate placement of solder and a die onto wire. Solder dots (lead-free, antimony-free rosinbased solder paste, part number 7024454: Nordson EFD, Dunstable, UK) were placed onto copper wire using a Fisnar robot, model F5200N (Intertronics, Kidlington, UK) holding a Nordson solder dispenser operated by a Nordson EFD Ultimus I precision fluid dispenser (Nordson EFD, Dunstable, UK). A pick and place device (QM1100: SMT Max, Chino California,

a)
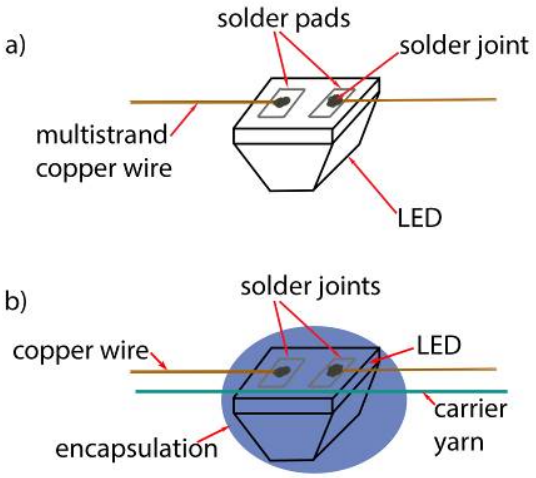

c)

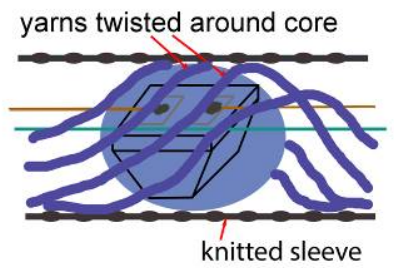

Fig. 2. Schematic cross section showing: a) LED soldered to wire; b) Carrier yarn included in encapsulation of LED; c) Addition of yarns twisted around the core and knitted sleeve 
USA) was then used to place the die onto the solder dots. An ATN lightbeam spot soldering system with IR-lamp (ATN LBS-G400, Berlin, Germany) was moved into place by the robot to apply heat, soldering the LED to the wire. The shortcircuit between the two terminals of the LED was then removed. A BTSR winding feeder (V14.052.1.0: BTSR, Olgiata Olona, Italy) and stepper motor (Vexta 2-phase model PK268-01A: Oriental Motor Co. Ltd., Japan) were used to tension and move the wire as more LEDs were soldered to the wire. Fig 3 shows the assembly of machinery used to carry out this process, alongside photographs of E-yarn at each stage of production.

\section{B. II. Creating a micropod around the package die}

This stage of the E-yarn production process involved the addition of a strong carrier fiber at the same time as accurate placement of a resin micropod: A Vectran ${ }^{\mathrm{TM}}$ carrier yarn was placed alongside the copper wire. The Vectran ${ }^{\mathrm{TM}}$ and copper wire with the soldered LED were moved into a silicone rubber cylindrical mould, where a polymer resin was placed around the LED. The resin was cured using a Bluewave ${ }^{\circledR}$ QX4 ${ }^{\mathrm{TM}} \mathrm{UV}$ curing system (Intertronics, Kidlington, UK). The machinery used for this stage of manufacture is shown in fig 4.

\section{III. Wrapping yarn around the wire and carrier fiber}

The carrier yarn and wire with attached LEDs were wrapped with three strands of cotton yarn (NM 30/1*2 Davidoff: Boyar Textile, Istanbul, Turkey) using an Agteks DirecTwist 2B6 machine (Agteks, Istanbul, Turkey) as shown on the left in fig 5. This machine was designed for use with standard textile yarns, so a slow feed and speed of 10 metres per minute was chosen so that the Vectran ${ }^{\mathrm{TM}}$ and copper wire with attached micropods could be fed through without damage.

\section{IV. Creation of knitted sheath around the E-yarn}

The twisted yarn, wire and carrier yarn was passed through an MC-D small-diameter warp-knitting machine (RIUS, Barcelona, Spain) (on the right in fig 5) to create a knitted sheath and to add packing yarns around the construction. Polyester yarn was used (167/36: J. H. Ashworth and Son Ltd, Hyde, UK). This produced a completed E-yarn.

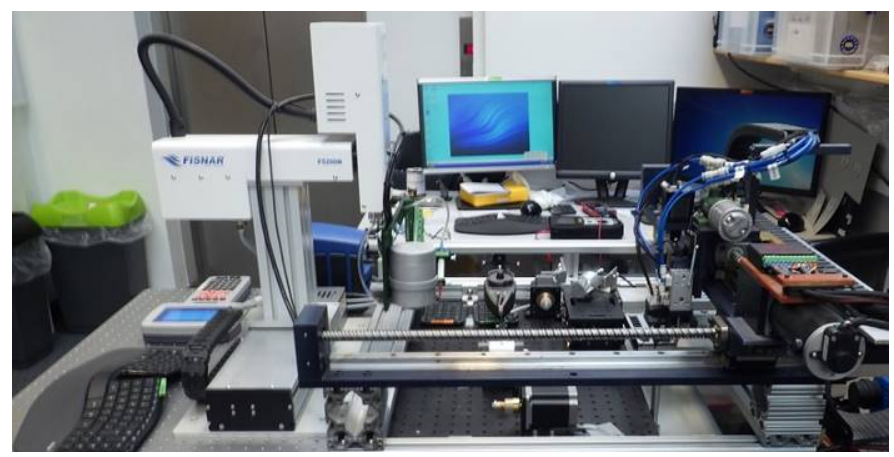

Fig. 3. The setup for soldering package dies to copper wire

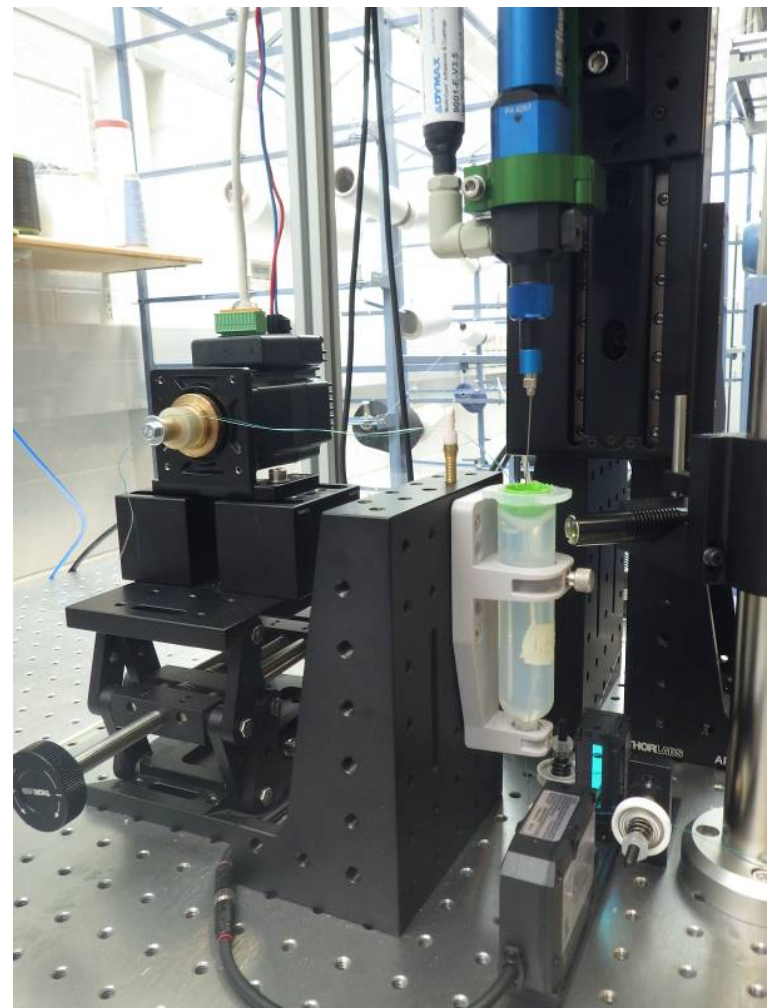

Fig. 4. The machinery for creation of a resin micropod around the package die
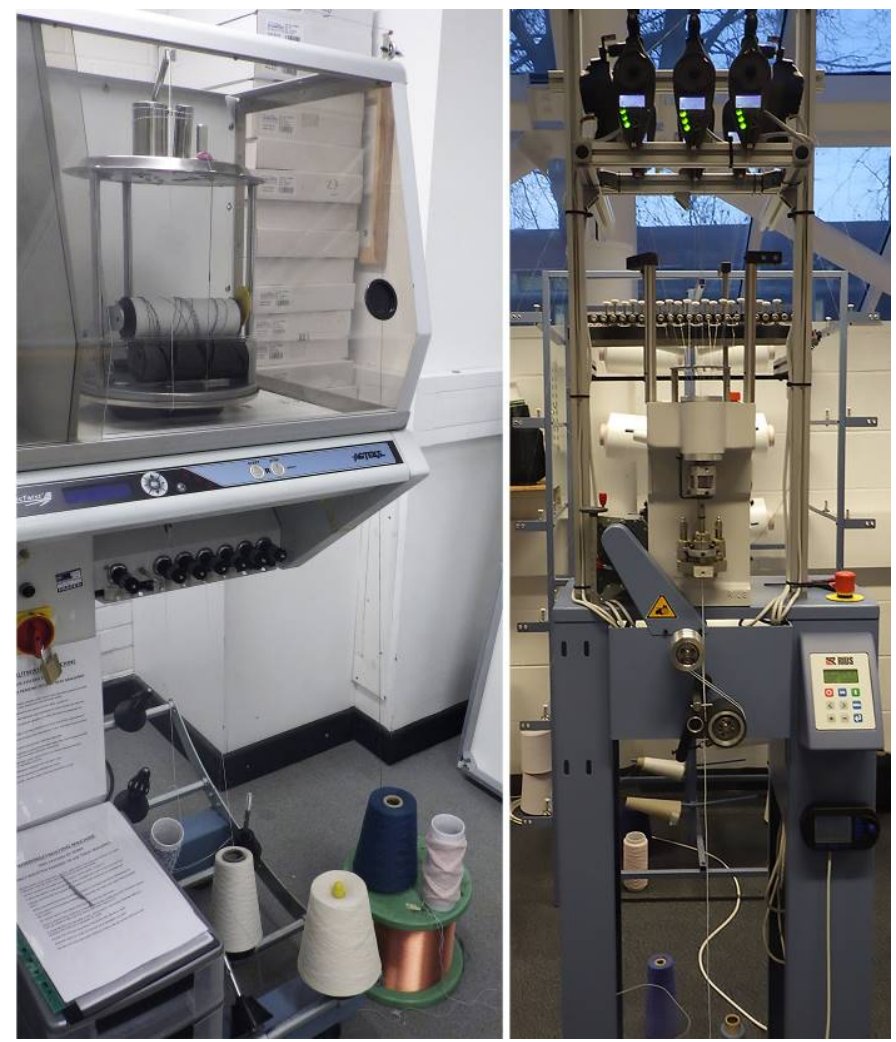

Fig. 5. Left: The Agteks yarn twisting machine. Right: The RIUS machine for creation of a knitted sleeve around the E-yarn 


\section{RESULTS AND RELIABILTIY}

The automated production process fashioned a flexible yarn of diameter $2 \mathrm{~mm}$ for incorporation into fabrics in subsequent knitting, weaving or embroidery processes. (Note that by using smaller dies, smaller yarn diameters can be produced.) The speed of the production process was increased from 60 to 90 minutes per die for the manual, craft process, to 6 minutes for the prototype, automated process. Figs 6 and 7 show magnified images of LEDs at the first two stages of the process. Fig 8 shows a completed LED yarn.

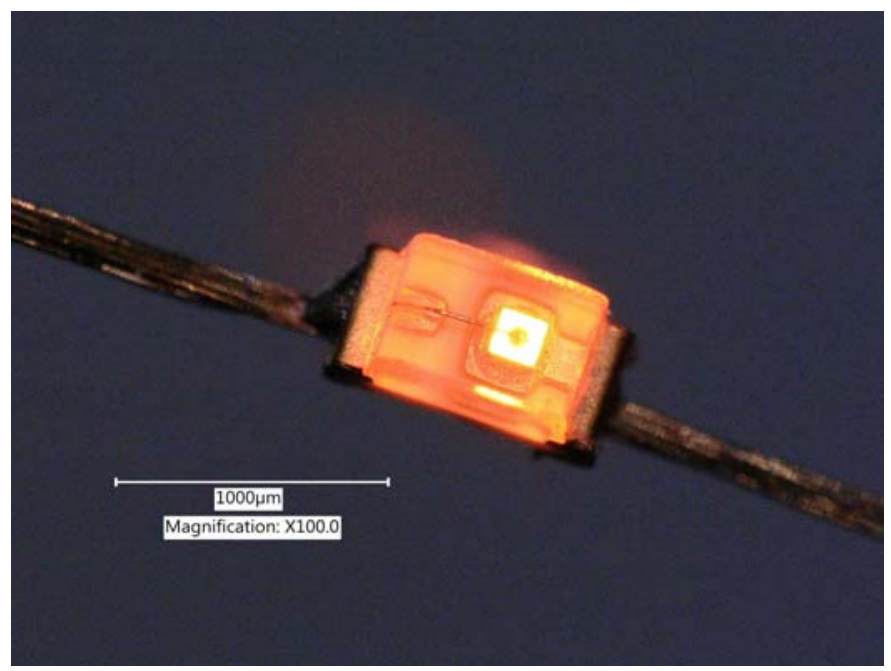

Fig. 6. LED soldered to copper wire after the first stage of the manufacturing process, magnified x 100

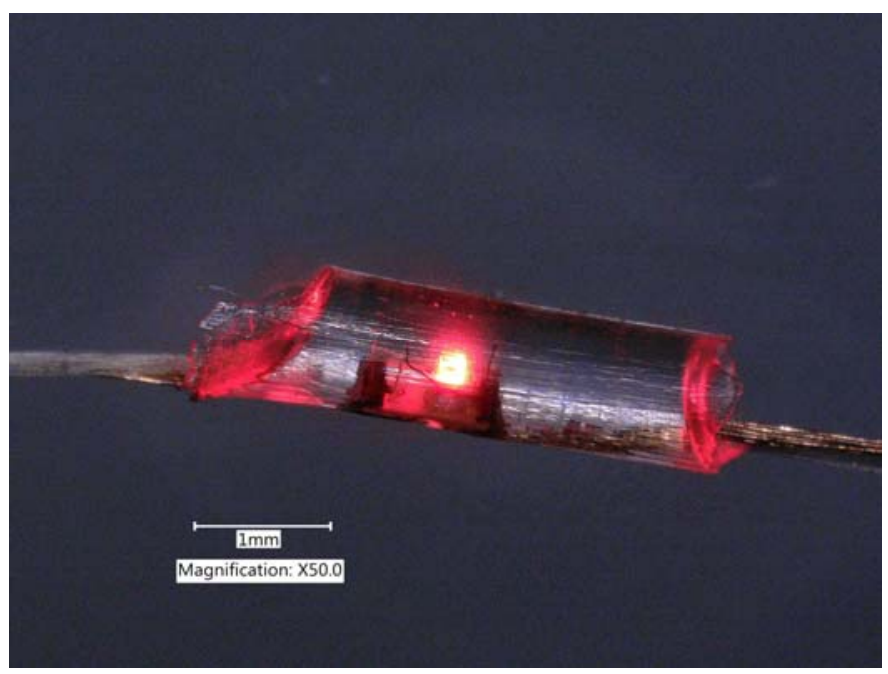

Fig. 7. Encapsulated LED after the second stage of the manufacturing process, magnified x 50

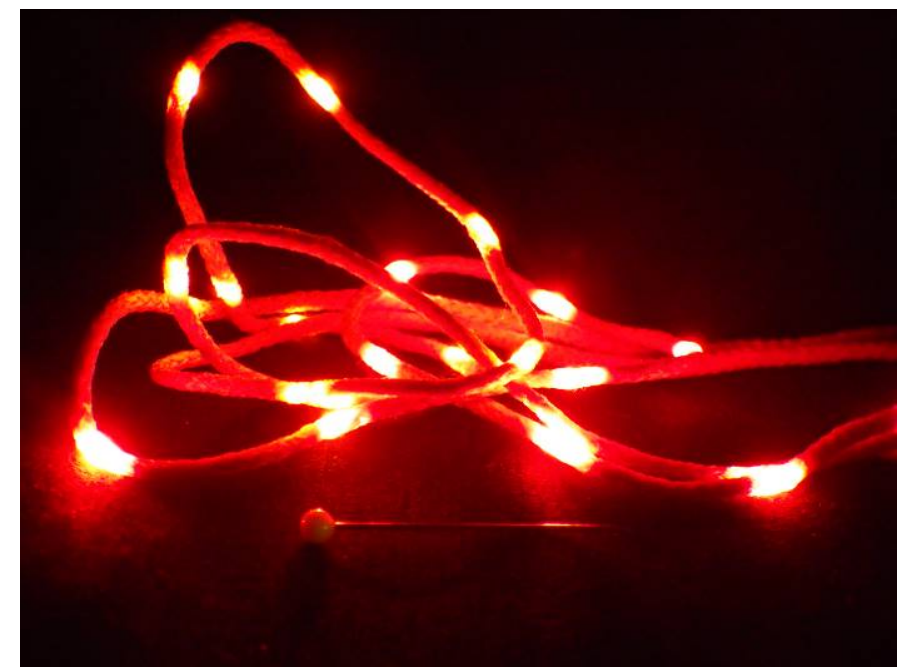

Fig. 8. Completed, illuminated LED yarn, shown next to a $30 \mathrm{~mm}$-long pin

The accuracy and reliability of LED placement was tested by creation of an E-yarn containing 9 groups of 5 LEDs, with the LEDs within each group to be spaced at $20 \mathrm{~mm}$. A voltage was applied, verifying that that each cluster of LEDs illuminated. The distances between LEDs were also measured. The results are shown in Fig. 9 below. The standard deviation in placement was $0.4 \mathrm{~mm}$, corresponding to $2 \%$ of the $20 \mathrm{~mm}$ spacing placement. The greatest variation on LED spacing was $0.7 \mathrm{~mm}$ from the target placement point.

\section{A. E-yarn strength}

The carrier fiber and outer, knitted sheath were expected to add tensile strength to E-yarn in the direction of its longest axis: along the length of the yarn. This was assessed by carrying out tensile tests on the copper wire; Vectran ${ }^{\mathrm{TM}}$ carrier yarn; knitted sheath; and completed E-yarn. These were tested on a zwickiline tensile tester (Z2.5, Zwick/Roell, Ulm, Germany) to ASTM E8 [12]. This testing standard was designed for use with metallic materials, so the test speed was reduced to $50 \mathrm{~mm} \mathrm{~min}^{-1}$ to assess the performance of the nonelastomeric textile yarns, as well as the copper wire under investigation. Fig 10 shows the results, with the greatest breaking force shown for the E-yarn knitted sheath $(95-111$ N).

The Vectran ${ }^{\mathrm{TM}}$ within the completed E-yarn was shown to reduce the final breaking force, and to decrease the elongation at break. The dotted line at the base of the inset graph shows the tension on the copper wire going through the initial stages of the E-yarn production process, compared with the forces on a copper wire tested to breakage, shown at the top of the inset. The forces applied during E-yarn manufacturing are 1\% of the breaking strain of the copper wire, indicating that the wire is not excessively tensioned during the yarn manufacturing process. 


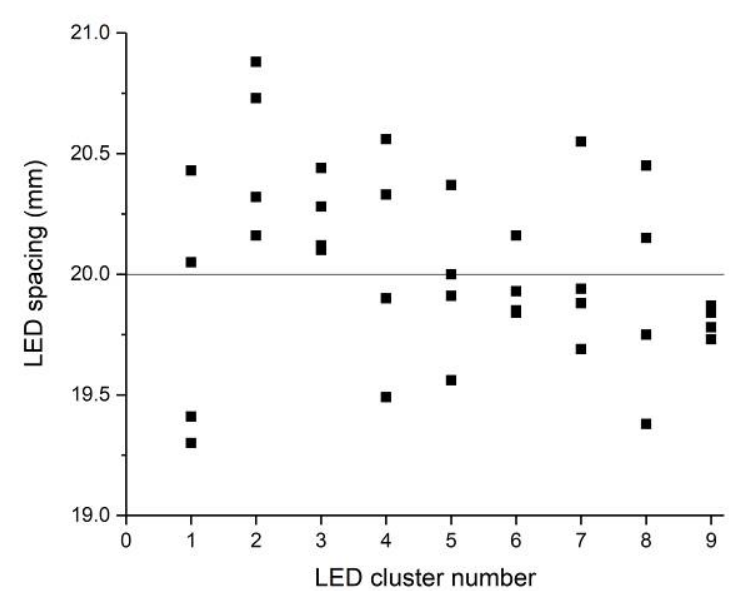

Fig. 9. Accuracy of soldered LED positioning on copper wire

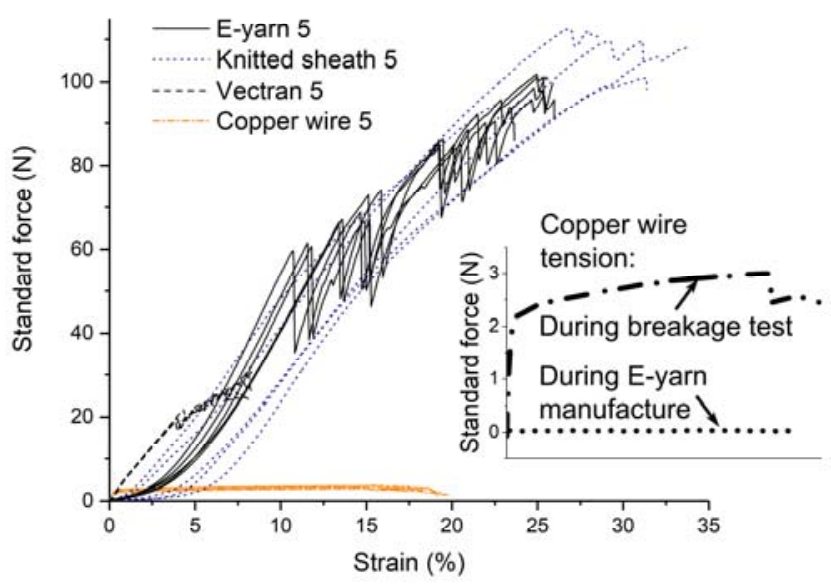

Fig. 10. Tensile test results for E-yarn and materials included within E-yarn. The inset compares the breaking force for copper wire with the tension in copper wire during E-yarn manufacture.

\section{FURTHER DEVELOPMENT}

The automated E-yarn production process that was described here involved the manual transfer of the partiallycompleted E-yarn from one stage of the process to the next. Spools of partially-completed E-yarn were passed between sets of machinery. A useful development would be to automate the transfer from one set of machinery to the next. Yarn tension and feed rates will require regulation during the transfers, to ensure optimal delivery to each stage of the production process.

Ongoing development of prototype textiles and garments containing E-yarns can now take place in parallel with developments in the E-yarn production process. This is likely to lead to requirements for new types of E-yarns, for which the production process can be adapted.

\section{CONCLUSION}

The automation of the E-yarn production process resulted in an increase of 1000 to $1500 \%$ in the speed of production of Eyarn. Accurate placement of dies was shown to be carried out with an error of $2 \%$. The process can now be developed further; including automation of the movement of partially-completed E-yarn between one set of machinery and the next. This will enable further increases in the speed of production of E-yarns, and lay the foundations towards a greater adoption of the technology.

\section{ACKNOWLEDGMENTS}

The authors acknowledge funding from EPSRC grant EP/M015149/1: Novel manufacturing methods for functional electronic textiles; and from Nottingham Trent University.

The authors would like to thank Dr. Arash Shahidi for carrying out tensile testing.

\section{REFERENCES}

[1] J. Hayward, "E-Textiles 2017-2027: Technologies, Markets, Players," IDTechEx, 2017. [Online]. Available: https://www.idtechex.com/research/reports/e-textiles-2017-2027technologies-markets-players-000522.asp. [Accessed: 08-Feb-2018].

[2] O. Tomico, L. Hallnäs, R.-H. Liang, and S. A. G. Wensveen, "Towards a Next Wave of Wearable and Fashionable Interactions," Int. J. Des., vol. 11, no. 3, pp. 1-6, 2017.

[3] T. K. Dias, "WO2016/038342 A1 Electronically functional yarns," Patent WO2016/038342 A1, 2016.

[4] DuPont, "DuPont TM Kapton ® Summary of Properties," 2017. [Online].

Available: http://www.dupont.com/content/dam/dupont/products-andservices/membranes-and-films/polyimde-films/documents/DEC-Kaptonsummary-of-properties.pdf. [Accessed: 19-Mar-2018].

[5] T. Dias and T. Hughes-Riley, "Electronically Functional Yarns Transform Wearable Device Industry," R\&D Magazine, vol. 59, no. 2, 19-21.

[6] T. Hughes-Riley, P. Lugoda, T. Dias, C. Trabi, and R. Morris, "A Study of Thermistor Performance within a Textile Structure," Sensors, vol. 17, no. 8, p. 1804, Aug. 2017.

[7] J. LaDou, "Printed circuit board industry," Int. J. Hyg. Environ. Health, vol. 209, no. 3, pp. 211-219, May 2006.

[8] Jianzhi Li, P. Shrivastava, Zong Gao, and Hong-Chao Zhang, "Printed circuit board recycling: a state-of-the-art survey," IEEE Trans. Electron. Packag. Manuf., vol. 27, no. 1, pp. 33-42, Jan. 2004.

[9] A. C. Siegel, S. T. Phillips, M. D. Dickey, N. Lu, Z. Suo, and G. M. Whitesides, "Foldable Printed Circuit Boards on Paper Substrates," Adv. Funct. Mater., vol. 20, no. 1, pp. 28-35, Jan. 2010.

[10] A. S. Rathnayake, "Development of the core technology for the creation of electronically-active, smart yarn," $\mathrm{PhD}$ thesis, Nottingham Trent University, Nottingham, UK, 2015.

[11] S. C.-Y. Lu and A. Liu, "Abductive reasoning for design synthesis," CIRP Ann. - Manuf. Technol., vol. 61, no. 1, pp. 143-146, 2012.

[12] ASTM, "ASTM E8/E8M - 16A Standard Test Methods for Tension Testing of Metallic Materials." ASTM Standard, 2016. 